\title{
Mirror structures above and below the linear instability threshold: Cluster observations, fluid model and hybrid simulations
}

\author{
V. Génot ${ }^{1}$, E. Budnik ${ }^{2}$, P. Hellinger ${ }^{3}$, T. Passot ${ }^{4}$, G. Belmont ${ }^{5}$, P. M. Trávníček ${ }^{3}$, P.-L. Sulem ${ }^{4}$, E. Lucek $^{6}$, and \\ I. Dandouras ${ }^{1}$ \\ ${ }^{1}$ CESR, Université de Toulouse (UPS) \& CNRS (UMR5187), Toulouse, France \\ ${ }^{2}$ Noveltis, 2 Avenue de l'Europe, 31520 Ramonville Saint-Agne, France \\ ${ }^{3}$ Institute of Atmospheric Physics and Astronomical Institute, AS CR, Prague, Czech Republic \\ ${ }^{4}$ CNRS, Observatoire de la Côte d'Azur, Nice, France \\ ${ }^{5}$ CETP-CNRS, Vélizy, France \\ ${ }^{6}$ Space and Atmospheric Physics Group, Blackett Laboratory, Imperial College, London, UK
}

Received: 23 May 2008 - Revised: 26 November 2008 - Accepted: 7 January 2009 - Published: 6 February 2009

\begin{abstract}
Using 5 years of Cluster data, we present a detailed statistical analysis of magnetic fluctuations associated with mirror structures in the magnetosheath. We especially focus on the shape of these fluctuations which, in addition to quasi-sinusoidal forms, also display deep holes and high peaks. The occurrence frequency and the most probable location of the various types of structures is discussed, together with their relation to local plasma parameters. While these properties have previously been correlated to the $\beta$ of the plasma, we emphasize here the influence of the distance to the linear mirror instability threshold. This enables us to interpret the observations of mirror structures in a stable plasma in terms of bistability and subcritical bifurcation. The data analysis is supplemented by the prediction of a quasistatic anisotropic MHD model and hybrid numerical simulations in an expanding box aimed at mimicking the magnetosheath plasma. This leads us to suggest a scenario for the formation and evolution of mirror structures.
\end{abstract}

Keywords. Magnetospheric physics (Magnetosheath; Plasma waves and instabilities) - Space plasma physics (Waves and instabilities)

\section{Introduction}

In a magnetized plasma, mirror modes and mirror structures are slowly evolving states, in which the magnetic fluctuations are compressible and in approximate pressure balance, lead-

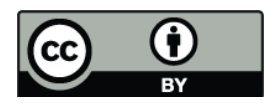

Correspondence to: V. Génot (vincent.genot@cesr.fr) ing to anti-correlated magnetic and plasma pressure (density) fluctuations. They are non-propagating in the rest frame of the plasma, when the later is homogeneous, but a drift velocity can result for example from variations of the ambiant field. They differ from the slow MHD modes and structures by the fact that the quasi-equilibrium is mainly based on the temperature anisotropy and not on a slow propagation. The time evolution of the linear mode can correspond either to an increase or to a decrease of the fluctuation magnitude (i.e. to instability or damping), depending on the value of the zero order anisotropy with respect to a threshold value, for which the temperature perpendicular to the ambiant magnetic field is larger than the parallel one. The nonlinear structures may be due to the nonlinear evolution of the instability, but the linear threshold value has anyway a pivotal role in the physics. To avoid any confusion, the terminology adopted in relation with mirror mode physics must be specified. In the following and in agreement with the common use of the literature, we shall use the terms "mode" and "wave" in relation with the solution of the linearized kinetic equations in the lowfrequency regime. We shall call "mirror structures" what we consider to be the nonlinear extension of the linear mirror mode. The object of the present paper is to investigate how these structures look like and how they form.

While one-spacecraft data were not sufficient to properly estimate the typical scales, two-spacecraft (Fazakerley and Southwood, 1994) and then multi-spacecraft analysis have shown that mirror modes are elongated in a direction making a small angle with the ambient magnetic field (Lucek et al., 2001; Walker et al., 2002; Horbury et al., 2004; Sahraoui et al., 2006). Furthermore, the magnetic fluctuations within these structures are often observed

Published by Copernicus Publications on behalf of the European Geosciences Union. 
to be far from being sinusoidal and are rather displayed as trains of large-amplitude holes or peaks, and in some instances solitary structures. The morphology of mirror structures is in fact a long standing issue. Magnetic depressions (holes) or enhancements (peaks) were observed in temperature anisotropic, high $\beta$ magnetosheath plasmas of a variety of celestial bodies which provide a natural location for mirror instability. Trains of magnetic holes and peaks were observed by Voyager 1 in the heliosheath and interpreted as mirror structures (Burlaga et al., 2006, 2007; Génot, 2008). Liu et al. (2006) (see also references therein) observed mirror modes ahead of interplanetary coronal mass ejections (ICME) preceded by shocks. Magnetic fluctuations mostly appear as large holes in regions where the plasma is marginally unstable with respect to the linear instability. These observations exhibit the presence of a plasma depletion layer (PDL) resembling those of planetary magnetosheaths. For Saturn, Voyager observations made on the dayside were reported by Bavassano Cattaneo et al. (1998). The authors track the evolution of mirror structures from a quasi-perpendicular bow shock to the magnetopause. The observed structures evolve from quasi-sinusoidal waves to non-periodic structures, consisting of both magnetic peaks and holes, and, finally, to holes in the PDL close to the magnetopause. For Jupiter, spiky structures were reported for the first time by Erdös and Balogh (1996). A more recent and thorough survey of all the Jovian magnetometer data (Joy et al., 2006) shows that $33 \%$ of the observed structures are either holes or peaks, with peaks primarily observed on the day side in the high $\beta$ plasma of the middle magnetosheath, and holes mostly in low $\beta$ plasma near the magnetopause and on the flanks. In the Earth context, observations of mirror structures are available since the early measurements by Explorer 12 (Kaufmann et al., 1970); Leckband et al. (1995) using AMPTE-UKS termed the observed peaks "monolithic" structures. Equator-S observations of peaks and holes have been reported by Lucek et al. (1999) but the spatial distribution of these structures was not investigated further and no information on the plasma parameters are available for this spacecraft. Later on, when studying kinetic aspects of the mirror mode, Génot et al. (2001) listed a number of mirror mode events observed by AMPTE-UKS \& IRM and some of them were tagged "spiky up" and "spiky down" by contrast with the more sinusoidal ones. However, in this case also, it was difficult to draw any conclusion on the occurrence conditions, as the plasma data were scarce in these intervals, except for the 21 September 1984 13:13-13:36 IRM event which exhibits very deep magnetic holes $(\delta B / B \simeq 0.6)$ in a mirror stable region, a peculiarity which will be addressed in the following.

For the solar wind, Winterhalter et al. (1994) made a survey of magnetic holes observed by Ulysses and examined their possible relationship with mirror instability. They found that holes tend to occur in the interaction regions where fast streams overtake the ambient solar wind and the plasma is marginally stable. Using the same detection criterion on a larger set of data from Ulysses, Helios $1 \& 2$ and Voyager 2, Sperveslage et al. (2000) reinterpreted magnetic holes as a combination of both mirror modes and solitons. Mirror mode dynamics is indeed not the only theory proposed to account for the observed magnetic peaks and holes. For instance Stasiewicz (2004a,b) (see also references therein) interpreted Cluster observations in term of trains of slow magnetosonic solitons, which are nonlinear wave solutions of Hall-MHD equations, an approach initiated by Baumgärtel et al. (1997) and Baumgärtel (1999).

Several models in connection with observations have been proposed (Joy et al., 2006; Tátrallyay and Erdös, 2002) to explain the evolution of mirror modes from the bow shock to the magnetopause, whereas computational (Pantellini, 1998; Baumgärtel et al., 2003) or theoretical (Kivelson and Southwood, 1996) works investigated how these structures could be formed. In the context of Jupiter's magnetosheath, Joy et al. (2006) follow this last model and synthesize the picture by noting that magnetic peaks could be the signature of nonlinear saturation of mirror modes; hole structures are also observed and interpreted as collapsing structures in a plasma near the linear mirror threshold, a phase termed stochastic decay as individual structures are supposed to decay at different rates. A relation between the shape of the structures and the local plasma parameters is suggested: peaks are mostly observed in high $\beta$ regions, and holes in low $\beta$ regions in agreement with the model by Pantellini (1998). In the model of Kivelson and Southwood (1996), the return to marginal stability is made possible by the formation of magnetic fluctuations with slight compressions and large depressions, which would favor peaks.

The formation mechanism for both magnetic peaks and holes, and in particular their relation with the mirror instability, is however not fully understood. Insight was given by observations by Erdös and Balogh (1996) in which the signature of a bistable behavior between region of high and low magnetic field was evidenced. To account for this observation, Baumgärtel (2001) constructed a fluid model with a double polytropic closure equation which revealed that magnetic holes can survive mirror stable plasma conditions, while peaks cannot. This is a signature of a bistability phenomenon which is related to the existence of a sub-critical bifurcation. In this paper, we rely on this concept to analyze mirror structures in the Earth magnetosheath with Cluster data. Through the use of both magnetic field and plasma data, we develop discriminating methods to identify mirror events among magnetosheath passes and to assign them a shape related to the skewness of the magnetic field distribution.

Observations lack the dynamical perspective and are affected by spatial/temporal effects. Therefore, in order to complement the observational approach, we discuss recent numerical and theoretical models that compare favorably with our observations. For this purpose, we consider hybrid 
particles-in-cell simulations of the Vlasov-Maxwell equations, developed to investigate the effect of a slow expansion on a magnetosheath-like plasma and low frequency turbulence (Trávnícek et al., 2007). This approach enables us to present a dynamical perspective on the mirror mode evolution and in particular to point out peak-to-hole transition, together with the phenomenon of bistability. We also rely on the theoretical work of Passot et al. (2006) to provide a basis to the bistability phenomenon. More recent theoretical works aimed to understand mirror mode dynamics include Borgogno et al. (2007); Kuznetsov et al. (2007a,b); Califano et al. (2008).

The paper is organized as follows. Section 2 presents the data selection and the way magnetosheath passes are identified. The methods we employ to characterize mirror structures are discussed in Sect. 3 and tested in Sect. 4 on a case study. Section 5 presents statistics on the structure locations and discusses correlations with in-situ parameters. Observational results are compared with numerical and theoretical models in Sect. 6. Our main results are summarized in the Conclusion.

\section{Data and magnetosheath model}

\subsection{Data}

Five years (1 February 2001 to 31 December 2005) of the Cluster mission are considered. Cluster 1 magnetic field (FGM, Balogh et al., 2001) and on-board calculated ion moments (from the HIA experiment on the CIS instrument, Rème et al., 2001) data are used at $4 \mathrm{~s}$ resolution. Based on the same dataset a statistical analysis of mirror mode occurrence (localisation, fluctuation amplitude) has recently been published by Génot et al. (2009). The results compare favorably with those of a study based on ten years of ISEE data (Verigin et al., 2006). Cluster orbital configuration is ideally designed to study the magnetosheath whose various regions are correctly sampled in our analysis, with a majority of events close to the magnetopause. A web-based version of the statistical analysis tool developed at CDPP (the French Plasma Physics Data Centre) and used in this study is available at the URL: cdpp-amda.cesr.fr. Access is granted upon request (mail to amda@cesr.fr).

\subsection{Magnetosheath identification}

The first step of our analysis is to determine whether Cluster is located in the magnetosheath. Data are analyzed by $5 \mathrm{~min}$ window: a delay procedure is applied to obtain associated solar wind and IMF parameters from ACE. Shock and magnetopause models, as described by Verigin et al. (2006) and Shue et al. (1997) respectively, are computed dynamically from these parameters in order to locate Cluster as "inside" or "outside" the magnetosheath.

\subsection{Fractional distance in the magnetosheath}

We will make use of the fractional distance

$F=\frac{r-r_{M P}}{r_{B S}-r_{M P}}$

introduced by Verigin et al. (2006) to normalize event positions in the magnetosheath. Here, $r$ is the Cluster geocentric distance, $r_{M P}$ the geocentric distance to the magnetopause (which is a function of the zenith angle, the solar wind ram pressure and IMF $B_{z}$ ) and $r_{B S}$ the geocentric distance to the bow shock (which is a function of the zenith and clock angles, the upstream Alfvén and Mach numbers and the angle between the solar wind velocity and IMF vectors). As a consequence $F=0$ at the magnetopause and $F=1$ at the bow shock.

\section{Mirror mode characterization}

Identification of mirror mode events has been a long standing issue. Both slow mode and mirror modes indeed display anticorrelated magnetic and density fluctuations. Moreover, both mirror and ion cyclotron modes develop in the presence of temperature anisotropy $\left(T_{\perp}>T_{\|}\right)$. It has nevertheless been predicted by Gary et al. (1993) and observed by Anderson et al. (1994) that due to the presence of heavier ions (mainly helium) in the magnetosheath, mirror instability dominates for $\beta>1$, which is the most common situation. This condition is confirmed by recent simulations by Trávnícek et al. (2007) $\left(\beta_{\|} \geq 0.35\right)$.

Various methods have been developed to discriminate between the various low-frequency modes and isolate mirror modes. They include transport ratio (Song et al., 1994; Denton et al., 1995), minimum variance analysis (Tátrallyay and Erdös, 2005), 2- and 4-spacecraft methods (Chisham et al., 1999; Génot et al., 2001; Balikhin et al., 2003; Horbury et al., 2004), $90^{\circ} \mathrm{B} / V_{z}$ phase difference (Lin et al., 1998). A comprehensive review of these approaches is given in Schwartz et al. (1996).

\subsection{Identification of mirror-like structures}

Magnetic field variations associated with mirror modes are almost linearly polarized in the direction of the ambient field. They may be of large amplitude (a few 10\%). From these characteristics, a criterion has been established which follows closely those used by Tátrallyay and Erdös (2005); Soucek et al. (2008); Génot et al. (2009); it requires two conditions:

- in order to select a linear polarization with field variations in directions close to that of the ambient magnetic field, the angle between the maximum variance direction and the mean magnetic field is prescribed to be smaller than $20^{\circ}$. 
- to prescribe relatively large amplitude, the variance of the field must be larger than $10 \%$.

In order to perform a statistical survey over 5 years of data, we use relatively low resolution data (4s), which limits the lower sampled mirror event size to $8 \mathrm{~s}$. From a 2 month survey with high resolution Cluster data, Soucek et al. (2008) found that mirror events were distributed as a bell-shaped distribution with $98 \%$ of events falling into the $4 \mathrm{~s}-24 \mathrm{~s}$ interval and with a mean of $12 \mathrm{~s}$. This shows that our data set is undersampled, as it misses events in the 4 to $8 \mathrm{~s}$ length. This corresponds to the structures with a smaller spatial scale (of the order of 10 local Larmor radii) even though flow velocity and field geometry do affect the observed temporal scales. The way the scale of events affects the statistics still remains to be studied with higher resolution data.

The above criterion is applied to all 5 min magnetosheath intervals selected in Sect. 2.1. The mean magnetic field is calculated on $10 \mathrm{~min}$ window, and the Minimum Variance Analysis (MVA) (Sonnerup and Cahill, 1967) is performed on 5 min windows. The sensitivity of the results was tested against the variation of these time windows and revealed no major difference. At this stage, we do not make any restriction on the values of plasma parameters, as we are interested in mirror-like structures appearing above as well as below the linear mirror instability threshold given by Eq. (2). No constraint has been imposed on the eigenvalues $\lambda$ resulting from the MVA. Indeed, as noted by Génot et al. (2001), mirror modes are more frequently observed with an elliptic polarization than with the linear polarization predicted by the linear theory for plane waves. We checked that restraining our data set to linearly polarized events (for instance with the condition: $\lambda_{\text {int }} / \lambda_{\max } \leq 0.2$ and $\lambda_{\min } / \lambda_{\text {int }} \geq 0.3$ ) does not significantly alter the conclusions. Note that Tátrallyay and Erdös (2005) supplement their criterion with a condition on the symmetry of the structures which essentially selects magnetic depressions. Therefore, only "dip" or "hole" mirror structures are discussed in that paper. Automatic detection of data patterns is a difficult task and it is possible that compressional structures other than mirror modes (e.g. quasiperpendicular shocks) may be retained by our algorithm. It is however difficult to evaluate the proportion of such misinterpreted events.

\subsection{Mirror condition}

The general form of the threshold condition for the mirror instability in a plasma composed of electrons, protons and alpha particules $\left(\mathrm{He}^{2+}\right)$ with bi-Maxwellian distribution functions and evaluated in the low-frequency,long-wavelength limit of the Vlasov-Maxwell equations reads (Stix, 1962; Hall, 1979; Hellinger, 2007)

$$
\sum_{s=p, \alpha, e} \beta_{s \perp}\left(\frac{T_{s \perp}}{T_{s \|}}-1\right)>1+\frac{\left(\sum_{s=p, \alpha, e} n_{s} q_{s} \frac{T_{s \perp}}{T_{s \|}}\right)^{2}}{2 \sum_{s=p, \alpha, e} \frac{\left(n_{s} q_{s}\right)^{2}}{\beta_{s \|}}}
$$

where $n, q$ and $T$ are the density, the charge and the temperature, respectively and $\beta=2 \mu_{0} n k T / B^{2}$. Subscripts $\perp$ and $\|$ stands for the directions with respect to the ambient magnetic field $B$, and $e, p$ and $\alpha$ for electrons, protons and alpha respectively. In the case of cold electrons, the previous condition reduces to

$\beta_{p \perp}\left(\frac{T_{p \perp}}{T_{p \|}}-1\right)+\beta_{\alpha \perp}\left(\frac{T_{\alpha \perp}}{T_{\alpha \|}}-1\right)>1$,

In the detector used in this study (HIA, see Sect. 2) ions are binned according to their energy per charge ratio. Therefore proton and alpha particles are generally mixed and moments are averages over these populations (we use the subscript $i$ for ions). In order to characterize the plasma with respect to the mirror instability, we thus define the mirror parameter by

$C_{M}=\beta_{i \perp}\left(\frac{T_{i \perp}}{T_{i \|}}-1\right)$.

$C_{M}-1$ is then the distance to threshold. From Eq. (3), the condition $C_{M}<1\left(C_{M}>1\right)$ corresponds to mirror stable (unstable) plasma, while $C_{M}=1$ refers to marginal stability, for which the linear growth rate is zero.

Although ion species are not separately discriminated we evaluated the error introduced by computing averaged ion moments rather than individual ones. With the typical values $T_{\alpha \|} / T_{p \|}=4, n_{\alpha} / n_{p}=0.04$ and an alpha anisotropy equal to the proton one we obtain $\sim 6 \%$ overestimation of the $C_{M}$ value. This figure increases up to $10 \%$ for large alpha to proton anisotropy and/or density ratios. Such an overestimation is however still within the overall measurement uncertainties.

As mentioned at the beginning of this section, previous equations apply for bi-Maxwellian plasmas only. Linear theory of the mirror instability with arbitrary distribution functions has been considered in Shapiro and Shevchenko (1964); Pokhotelov et al. (2002); Hellinger (2007); Califano et al. (2008). In particular, simulations by Califano et al. (2008) show that near threshold initially Gaussian distributions are flattened by quasi-linear effects for small velocities, which requires the use of the full criterion

$$
-\frac{m_{p}}{p_{B}} \int \frac{v_{\perp}^{4}}{4} \frac{\partial f}{\partial v_{\|}^{2}} d^{3} v-\beta_{\perp}-1>0
$$

where $f$ is the distribution function, $p_{B}$ is the magnetic pressure and $m_{p}$ the proton mass.

\subsection{Peaks and holes identification}

An automatized detection of structures is a prerequisite for any long term analysis. Tátrallyay and Erdös (2005), analyzing 10 years of ISEE-1, used an algorithm searching 

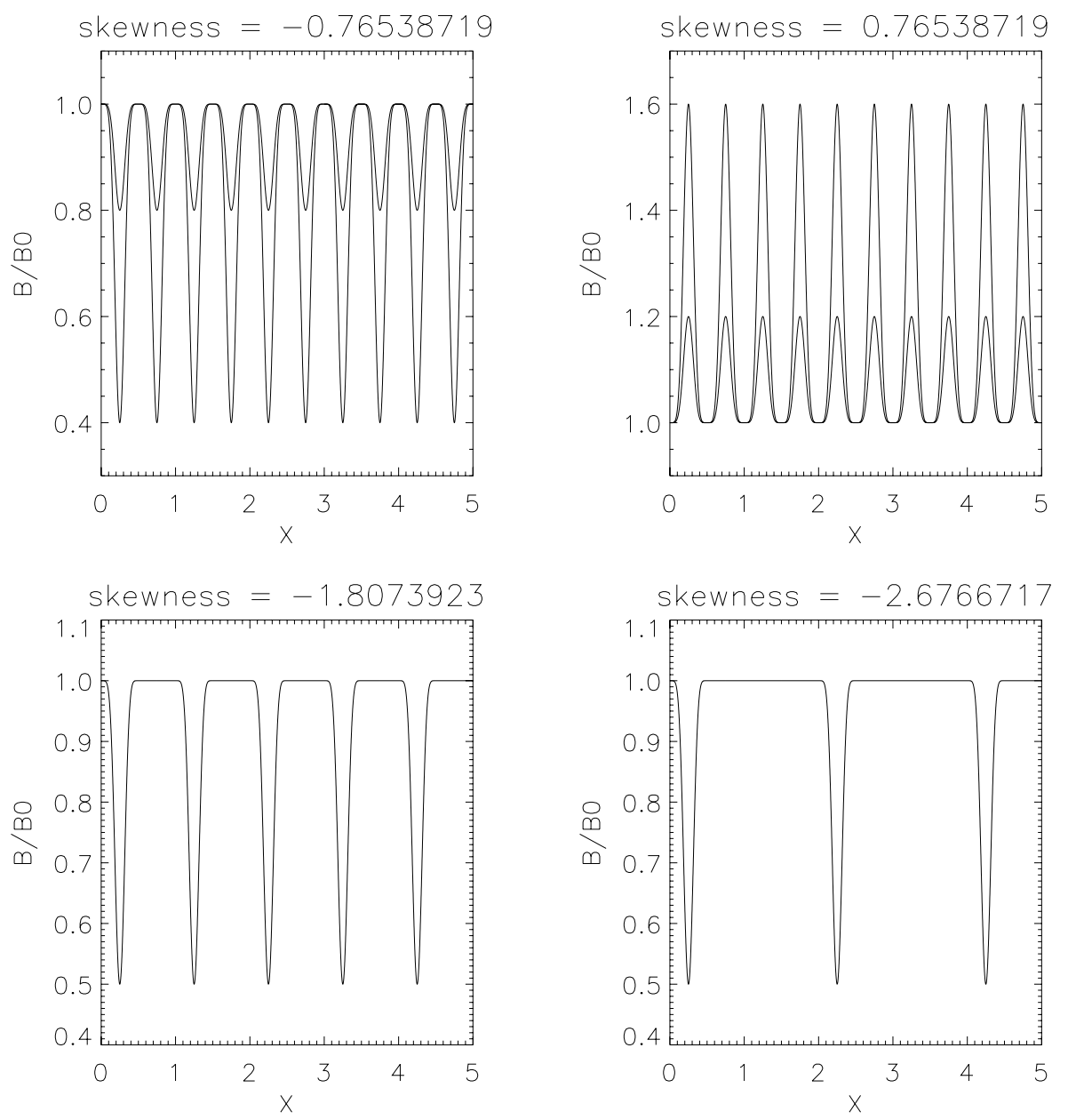

Fig. 1. Skewnesses of composite peak and hole structures obtained by varying the fluctuation amplitudes (top panels) and the number of structures on a given interval (bottom panels). Abscissae are arbitrary.

for symmetrical shapes of magnetic depressions. Joy et al. (2006) proposed a statistical method based on the determination of the background magnetic field level. For our analysis, we consider the list of 5 min magnetosheath intervals in which mirror-like structures were detected by the algorithm steps described earlier; we then compute the skewness (i.e. the normalized third moment) of the distribution of the magnetic fluctuations $(\delta B=B-<B>$, where the average is taken on a $10 \mathrm{~min}$ window). A positive (negative) value reflects a distribution skewed towards higher (smaller) values, which corresponds to an interval dominated by peaks (holes). A vanishing or small value corresponds to sinusoidal-like (symmetric) fluctuations or alternatively to an interval composed of peaks and holes equally distributed. The latter regime is usually associated with transition periods. The method was proved to be efficient by showing it correctly captures peakor hole-filled intervals identified by visual inspection.

Simultaneously to our analysis, an alternative and somewhat more refined algorithm based on the peakness of the distribution was developed and used by Soucek et al. (2008),
The peakness is defined as the skewness of the time series representing the total wavelet content, between two chosen scales, of the original magnetic field fluctuations. In a way similarly to that of our method, negative (positive) peakness of a given interval corresponds to magnetic holes (peaks).

Figure 1 shows changes in the skewness value when fluctuations are varied. Top panels show that varying the amplitude $\delta B / B_{0}$ while preserving the same underlying shape does not affect the skewness whose sign is simply reversed when fluctuations are changed from holes (left, $B_{0}-|\delta B|$ ) to peaks (right, $B_{0}+|\delta B|$ ). On the bottom panels, the shape of the holes is the same as in the top panels, but five (left) and seven (right) holes are removed. Consequently the skewness decreases from -0.77 to -1.81 and -2.68 , respectively. This indicates that larger (smaller) values of the skewness are not related to higher (deeper) structures but to a larger deviation from the sinusoidal shape which also translates into the presence of more isolated structures. Analyzing the amplitude of these structures would require a more refined algorithm (see for instance Soucek et al., 2008). 


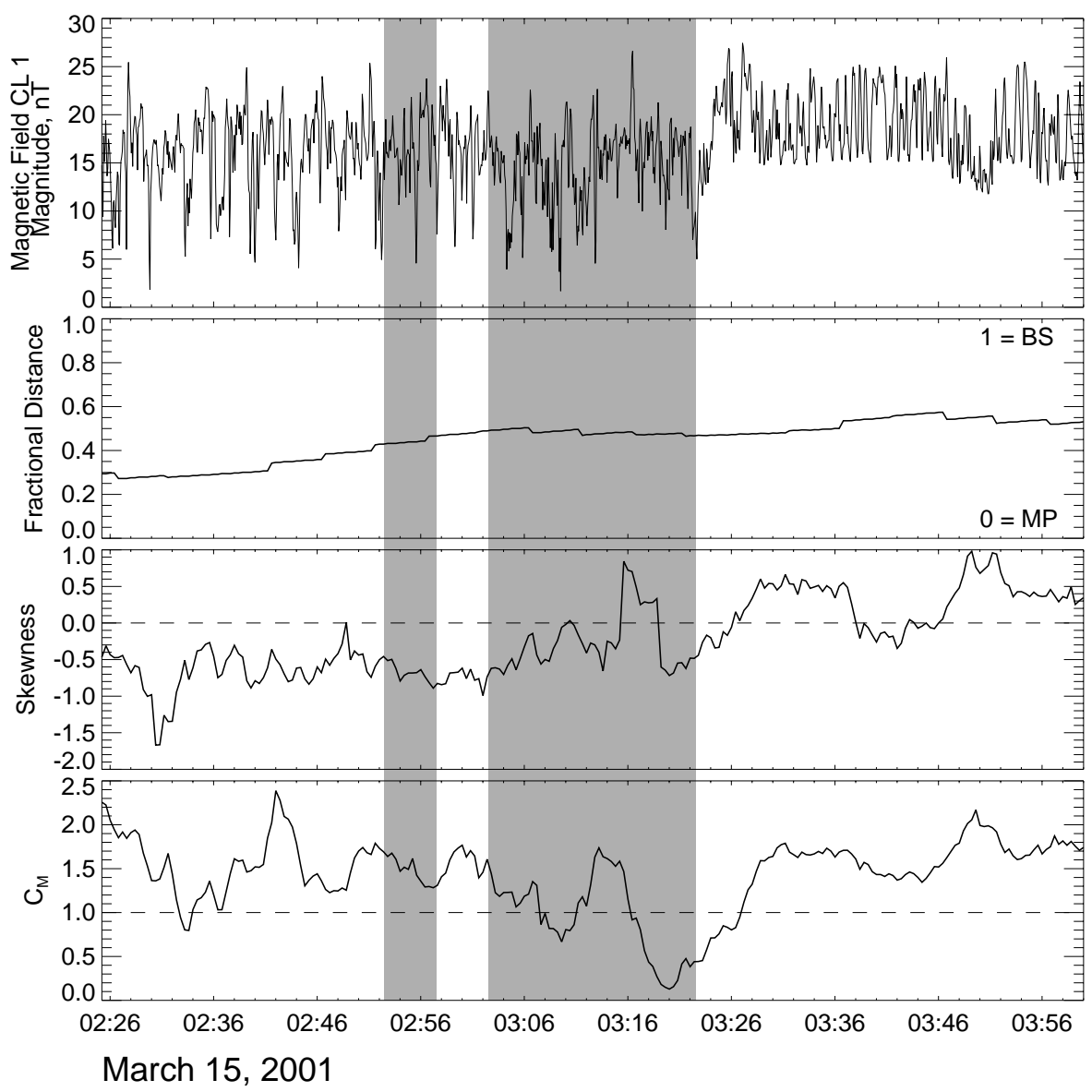

Fig. 2. Observations of mirror structures by Cluster 1 on 15 March 2001. From top to bottom: magnetic field amplitude, fractional distance $F$, skewness of magnetic fluctuations and mirror parameter $C_{M}$. The grey color codes windows of 5 min when mirror structures are not detected by the algorithm based on magnetic fluctuations.

\section{Case study: 15 March 2001}

In order to validate the above algorithm, we present in Fig. 2 observations which satisfy the mirror conditions on the magnetic field fluctuations and exhibit, during the course of a few hours, both holes and peaks, separated by a sharp transition. It appears that this transition is due to an abrupt change in the direction of the IMF, associated with a variation in the solar wind velocity. Data are from the Cluster and ACE satellites on 15 March 2001 from 02:23 to 04:00 (as recorded by Cluster), during which the conditions expressed above for mirror mode selection are almost totally fulfilled in each $5 \mathrm{~min}$ subinterval (grey color refers to the time intervals where they are not). The solar wind data are averaged on a 20-min window and the time delay between ACE and Cluster is about $3500 \mathrm{~s}$ until $03: 10$ and then steadily increases to $3700 \mathrm{~s}$ at 04:00. Cluster is mainly moving along the $+X_{\text {gse }}$ direction from $[10.0,1.2,9.0] R_{E}$ to $[12.9,0.2,8.6] R_{E}$, away from the magnetopause and towards the bow shock. Cluster satellites are separated by about $0.1 R_{E}$ and ACE is positioned at $[226.6,-37.6,1.3] R_{E}$. From visual inspection of the vari- ations of the magnetic field amplitude, one identifies a first interval consisting mainly of holes followed by a second interval of peaks. A sharp transition occurs at 03:24. We detail below these two sequences.

\subsection{Holes: 02:25-03:24}

We consider the interval 02:25-03:24 which presents a series of several contiguous 5 min mirror intervals, 02:25:0002:52:30 and 02:57:30-03:02:30, and non-mirror intervals (shaded on Fig. 2). When focusing on mirror intervals, the fractional distance $F$ evolves from 0.25 to 0.45 , confirming that the satellites are moving away from the magnetopause. The fractional distance is computed every $20 \mathrm{~s}$, and so are the distance to mirror threshold and the skewness (for which the distribution is taken on 5 min window). The skewness varies from 0 to -1.8 with a mean value around -0.5 : the magnetic field is indeed shaped as holes. A very deep isolated hole (skewness $=-1.8$ ) with a small magnetic field $(B=1.7 \mathrm{nT}, \delta B / B=0.9)$ and lasting about $20 \mathrm{~s}$ is observed at $02: 30$. Throughout this interval the plasma is mirror unstable 
$\left(C_{M}>1\right)$, except for a short period when the deep hole is observed. We shall come back to this apparent peculiarity in the course of the discussion.

\subsection{Peaks: 03:24-04:00}

In the second interval Cluster 1 observes mirror structures located in the middle magnetosheath $(0.45<F<0.55)$. They are mostly shaped as peaks during two intervals (during the first one, the skewness is close to 0.5 , while in the second one, it ranges between 0.5 and 1 ), separated by a mixed structures interval (skewness close to -0.15 ). The plasma is mirror unstable with $C_{M}>1.5$ in most of the interval.

The sharp transition between the two ranges, observed at 03:24, is due to IMF turning and decelerating solar wind. Indeed, ACE data shifted accordingly (not shown) exhibit a rotation in the magnetic field (from $30^{\circ}$ to $70^{\circ}$ for $\left(\widehat{B_{x}, B_{y}}\right)$ and from $0^{\circ}$ to $50^{\circ}$ for $\left.\left(\widehat{B_{x}, B_{z}}\right)\right)$ and a sharp decrease in the solar wind velocity from $390 \mathrm{~km} / \mathrm{s}$ to $370 \mathrm{~km} / \mathrm{s}$, exactly at this time. The transition between the two magnetosheath regions is therefore a spatial effect related to a fast change in the upstream solar wind forcing, resulting in the reconfiguration of associated shocks and local conditions of the magnetosheath.

\section{Statistical analysis}

Based on the methods described above for determining magnetosheath intervals, 5647 linearly polarized compressive mirror structures were isolated and classified as "holes" $(44 \%)$ or "peaks" $(30 \%)$ depending on the skewness sign when $\mid$ skewness $\mid>0.2$, or "either" $(26 \%)$ otherwise. "Either" means that the structures in the interval are either sinus-like or a mixture of holes and peaks. This somewhat arbitrary criterion was chosen from visual inspection. The question then arises of the statistics on hole/peak distributions, in relation with in-situ parameters.

\subsection{Relation with the plasma $\beta$}

In Fig. 3 , the skewness is plotted as a function of $\beta_{\|}$. For moderate $\beta_{\|}$, observations are gathered toward negative skewness, whereas large $\beta_{\|}(\geq 4)$ conditions favor positive skewness. This positive correlation gives a vanishing skewness for $\beta_{\|} \simeq 3.5$, in agreement with other observational studies (Erdös and Balogh, 1996; Bavassano Cattaneo et al., 1998; Joy et al., 2006; Soucek et al., 2008).

As temperature anisotropy is anti-correlated with $\beta_{\|}$ (Anderson et al., 1994; Gary and Lee, 1994; Fuselier et al., 1994), we expect and indeed observe (not shown) that smaller anisotropy conditions favor peaks. The anticorrelation in our data set is such that

$\frac{T_{\perp}}{T_{\|}}=1+\frac{a}{\beta_{\|}^{b}}$

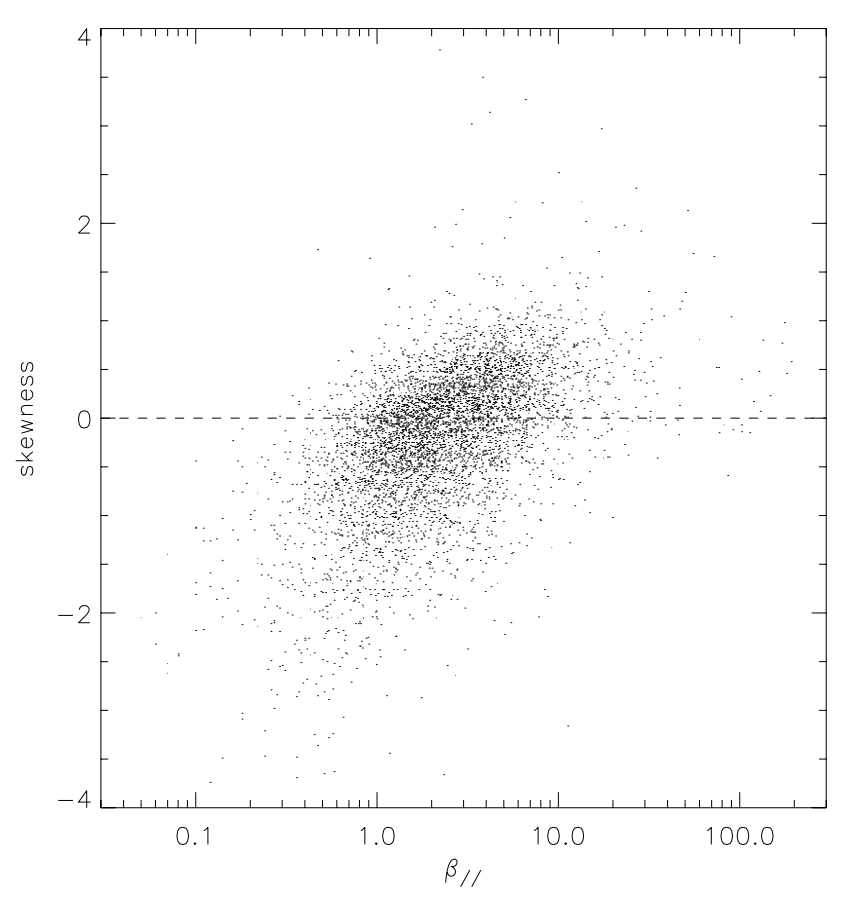

Fig. 3. Skewness as a function of $\beta_{\|}$for all mirror events detected in the period 1 February 2001-31 December 2005.

with $a=0.47$ and $b=0.56$ in rather good agreement with the values of $a=0.83$ and $b=0.58$ found for example by Fuselier et al. (1994) (for $\beta_{\|}>1$ ). This formal relation conveys the idea that the magnetosheath plasma remains in a marginal stable state with respect to the mirror instability and in a lesser measure with the ion cyclotron instability,as numerically demonstrated by Hellinger et al. (2003) and Trávnícek et al. (2007).

\subsection{Location}

To localize events in the magnetosheath, we use the fractional distance $F$ defined in Eq. (1). Let us first consider structures in a mirror unstable plasma $\left(C_{M}>1\right)$. On the right of Fig. 4, the average skewness is represented in each $F$-bin and shows that peaks are mostly observed in the middle magnetosheath $(F>0.3)$. Statistics close to the bow shock are poor and therefore negative values close to the shock are neither significant nor meaningful. A more detailed analysis (not shown) revealed that peaks are observed closer to the shock for increasing zenith angle; no dawn/dusk asymmetry is observed. Interestingly, if we now include mirror structures in unstable conditions ( $C_{M}>0$, left of Fig. 4), the average skewness close to the magnetopause is highly negative whereas it approaches zero in the middle magnetosheath. This is related to the fact that holes may be observed everywhere in the sheath, with a preference for the vicinity of the magnetopause and for mirror stable conditions. These findings are in excellent agreement with Fig. 9 of Joy et al. (2006) who 

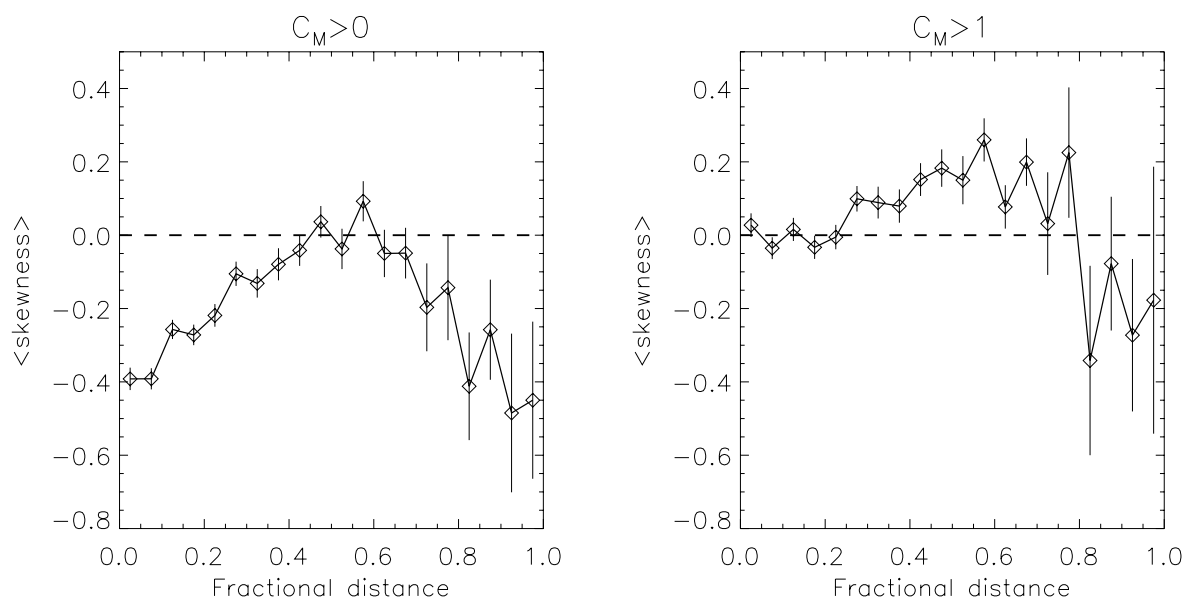

Fig. 4. Average skewness as a function of the fractional distance $F$ for $C_{M}>0$ (left) and $C_{M}>1$ (right). The error bars are proportional to $\sigma / \sqrt{N}$ where $\sigma$ is the standard deviation and $N$ is the number of mirror events in each $\Delta F=0.05$ bin $\left(N_{\min }=17\right.$ and $N_{\max }=709$ for $C_{M}>0$ and $N_{\min }=7$ and $N_{\max }=301$ for $C_{M}>1$ ).

showed that mirror structures are present everywhere in the (Jupiter) magnetosheath with peaks appearing preferentially in the middle sheath and holes close to the magnetopause at least for the day side magnetosheath data which represent most of our dataset. Further down the flank their observations suggest that holes fill the whole magnetosheath. Finally, the hole/peak localization in the magnetosheath is consistent with the $\beta$ spatial distribution ( $\beta$ decreases from the shock towards the magnetopause) and its dependence on the skewness discussed in the previous section.

\subsection{Influence of the distance to threshold}

Authors of similar observational studies (Erdös and Balogh, 1996; Joy et al., 2006) have emphasized the dependence of the mirror structure shape on the value of beta. However the way the mirror fluctuations evolve in a plasma is closely related to the distance to the mirror instability threshold. We show in the following that although this parameter corresponds to a linear threshold, it is also relevant to order data in the nonlinear regime for which mirror structures develop. This parameter is therefore a crucial variable for the physics of mirror mode dynamics. This also explains why this parameter is pivotal in all modeling approaches, be they analytical or computational, as discussed in the next section.

On Fig. 5, all events have been binned in intervals of the mirror parameter $C_{M}$ (Eq. 4). For each bin, the average skewness is computed as well as error bars. Transitions between peak/hole (skewness $=0$ ) and mirror stable/unstable $\left(C_{M}=1\right)$ have been over-plotted as dash lines. The insert shows that statistical significance is better (i.e. the number of observed events maximises) around $C_{M}=1$ which expresses the tendency of the magnetosheath plasma to be mostly observed in a marginally stable state with respect to the mirror instability (see for instance the observations reported in Anderson et al., 1994).

Inspection of this figure indicates that for increasing values of $C_{M}$, mirror magnetic fluctuations associated with unstable plasma conditions are shaped mostly as holes just above threshold, and as peaks far from it (the skewness increases up to 0.6 ). There is a transitional regime for which the skewness is close to zero (it vanishes for $C_{M} \simeq 1.4$ ) and which corresponds to sinusoidal variations or to a mixed regime of holes and peaks. All shapes of magnetic fluctuations may therefore be encountered under unstable plasma conditions. The striking observation comes from the stable plasma condition domain $\left(C_{M}<1\right)$. In this parameter range, very distinct magnetic hole structures are observed. The skewness varies from -0.3 to -0.7 , decreasing on average further off from threshold. It has to be stressed that this represents a general averaged trend, and some observations fall out the strict picture described above, as illustrated by error bars. These results are consistent with those of Soucek et al. (2008) where a similar positive correlation peakness-distance to threshold is described.

A bistability phenomenon can be invoked to explain these observations. This phenomenon expresses the fact that stable non zero solutions exist in a regime where the trivial solution is linearly stable. It is related to the presence of a subcritical bifurcation studied in details in Kuznetsov et al. (2007b). It was first modeled by Baumgärtel (2001) who performed fluid numerical simulations to explain the bistable behavior reported in Jovian magnetosheath observations by Erdös and Balogh (1996) (see also Baumgärtel et al., 2003). Existence of stable localized structures also exist in the context of anisotropic Hall-MHD (Stasiewicz, 2004a,b) in situations that are mirror stable. Symmetries of these solutions nevertheless differ from those of the structures observed in direct 
simulations of the Vlasov-Maxwell equations (Califano et al., 2008). Recently observations in the Earth magnetosheath with Cluster (Soucek et al., 2008; this study) have initiated an important theoretical effort to understand bistability, i.e. how mirror structures can survive in mirror stable conditions (Passot et al., 2006; Borgogno et al., 2007; Kuznetsov et al., 2007a; Califano et al., 2008). We shall come back to this issue in the next section devoted to comparison with models.

Note that, in binning the mirror parameter $C_{M}$ to display the average skewness in Fig. 5, the electron temperature effect is neglected. Taking this effect into account would lead to replace $C_{M}$ by $C_{M e}=C_{M}-\frac{1}{2} \frac{\left(\frac{T_{\perp}}{T_{\|}}-1\right)^{2}}{\frac{1}{\beta_{e}}+\frac{1}{\beta_{\|}}}$(see Eq. 2). Including the electron temperature term diminishes $C_{M}$ by $\sim 1$, $5,11,13 \%$ for $\beta_{e}=0.1,1,10,100$, respectively; this can be viewed as a small correction, comparable to the observational uncertainties and similar to the effect regarding ion measurements discussed in Sect. 3.2. Another effect concerns the electron anisotropy (in the magnetosheath $T_{\perp e} / T_{\| e} \gtrsim 1$ Gary et al. (2005); Masood and Schwartz (2008)). Both effects tend to reduce the mirror parameter (and then shift the curve of Fig. 5 to the left), but are not significant for our present discussion.

\subsection{Observations}

To illustrate the findings of the previous section, we chose two typical intervals displaying holes and peaks in their respective "natural environment". Mirror modes have been detected by our algorithm from the magnetic fluctuations throughout these intervals.

On 17 June 2002 (Fig. 6), holes are observed relatively close to the magnetopause $(0.12<F<0.19)$ with $\delta B / B$ up to 0.8 . The skewness varies from -0.15 down to -1.7 with the lower values corresponding to isolated depressions (at 15:17 for instance). The plasma is mostly mirror stable with two short excursions in mirror unstable conditions for which $C_{M}$ remains below 1.2. This is consistent with the fact that the peak/hole transition is seen for $C_{M} \simeq 1.5$ i.e. holes exist in stable conditions as well as in slightly unstable ones (close to threshold).

On 27 May 2005 (Fig. 7), peaks are observed close to the middle magnetosheath $(F \simeq 0.3)$ with relatively small enhancements $\delta B / B \simeq 0.3$. The skewness is quite variable whereas the plasma conditions remains highly mirrorunstable with $2.2<C_{M}<3.1$. By analogy with the simulation results which are presented in the next section, this observation may correspond to the saturated phase of the mirror instability with well developed peaks far from threshold.

\section{Discussion: comparison with theory and simulations}

Our final aim is to compare magnetosheath observations of mirror structures with theoretical models and numerical sim-

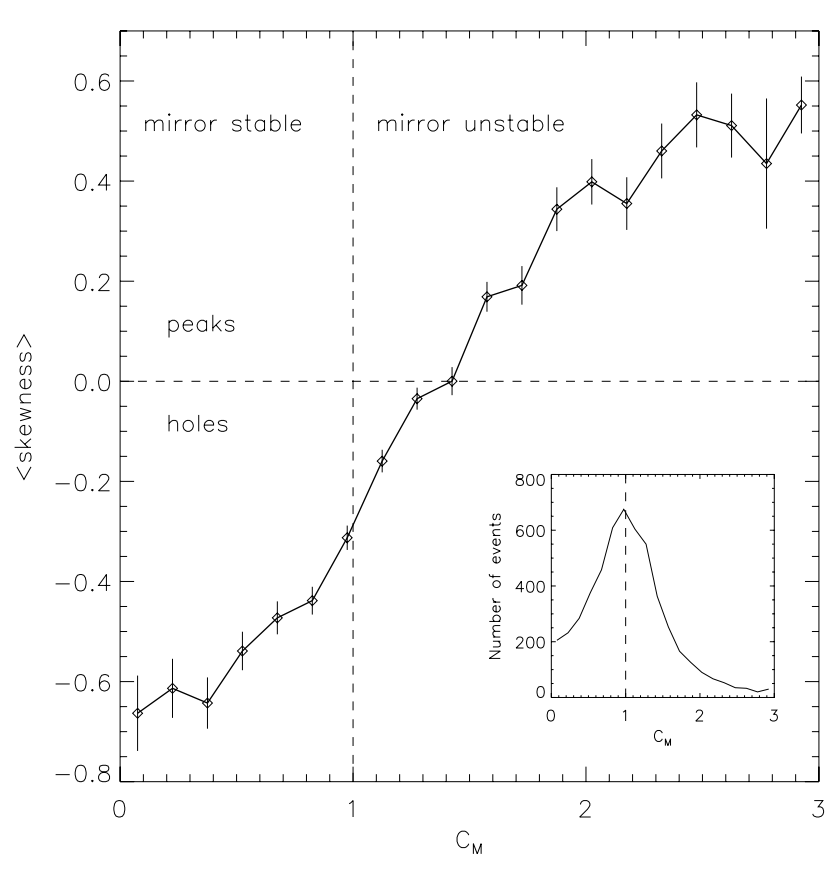

Fig. 5. Average skewness as a function of the mirror parameter $C_{M}$. The vertical dashed line delineates the mirror threshold. The horizontal dashed line delineates the region of predominance of holes (below) from the one of peaks (above). The error bars are proportional to $\sigma / \sqrt{N}$ where $\sigma$ is the standard deviation and $N$ is the number of mirror events in each $\Delta C_{M}=0.15$ bin $\left(N_{\min }=20\right.$ and $\left.N_{\max }=675\right)$. The insert shows the distribution of mirror events as a function of $C_{M}$ : the peak is observed for marginally stable conditions with respect to the mirror instability $\left(C_{M} \simeq 1\right)$.

ulations. For both these approaches, the key control parameter is the distance to the instability threshold.

Recently, a simple hydrodynamic description of pressure balanced magnetic structures has been proposed (Passot et al., 2006), based on anisotropic MHD equations supplemented by an equation of state suitable for the quasi-static regime. A similar equation of state has been kinetically derived by Constantinescu (2002). This model is able to accurately reproduce the mirror instability threshold. By minimizing the potential energy under the constraint of particle conservation and frozen-in magnetic field, stable solutions were obtained, in the form of magnetic holes at moderate $\beta$, or peaks when $\beta$ is larger, provided the angle $\theta_{k B}$ between the wave vector and the ambient field, together with the temperature anisotropy are sufficiently large. The model retaining no kinetic effects, the mirror structures correspond to piece-wise periodic non-linear solutions. An interesting point is that within this framework, the phenomenon of bi-stability is also observed, corresponding to the existence of stable nonlinear magnetic holes in a regime where the plasma is mirror stable. For the piece-wise solutions of the model, the skewness is equivalently replaced by a parameter $\lambda$ that measures the normalized length of the low magnetic field region (see Fig. 8 


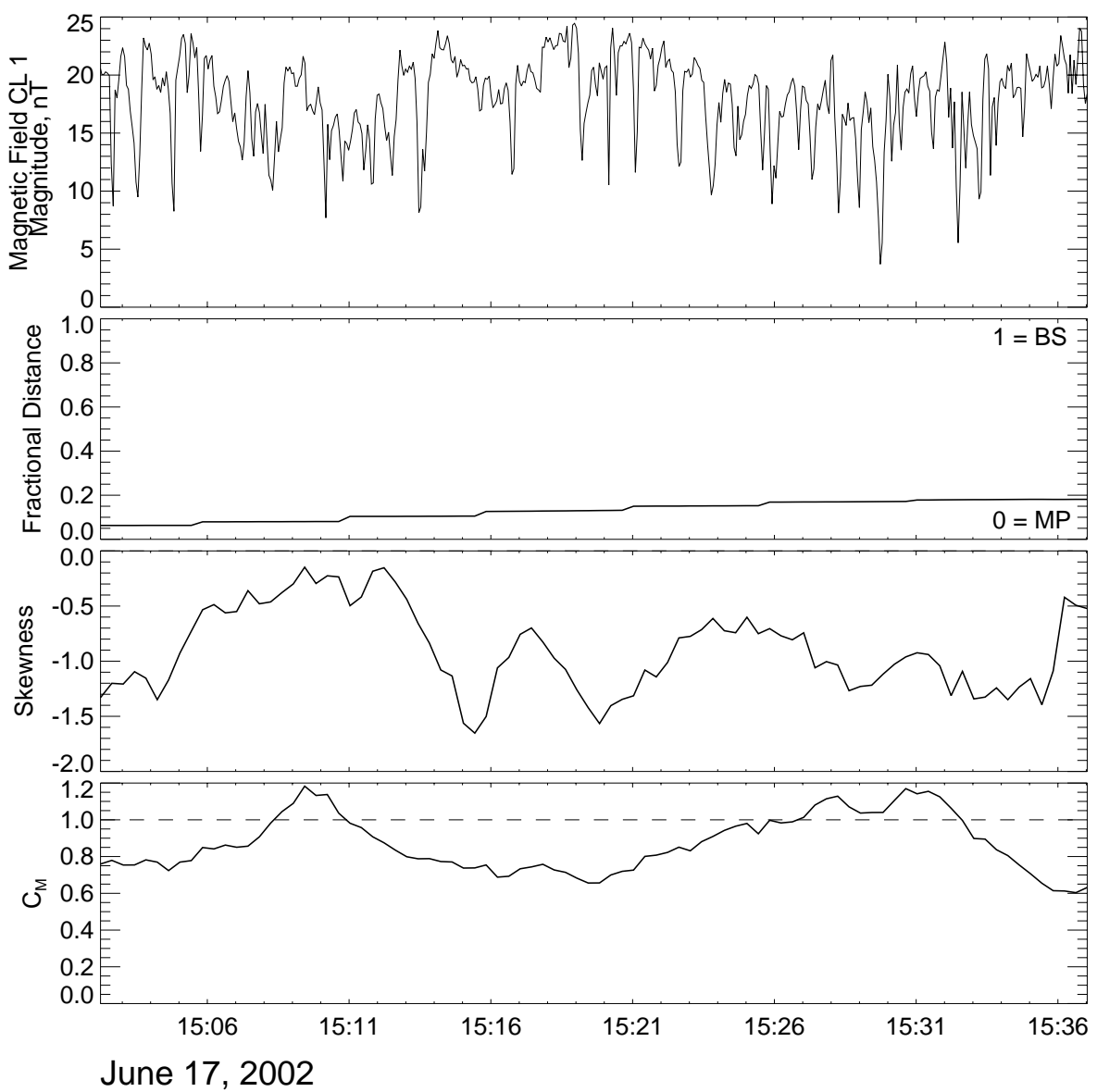

Fig. 6. Observations of magnetic holes by Cluster 1 on 17 June 2002 (same panels as in Fig. 1).

and Passot et al., 2006, for details on how it is computed). For $\lambda<0.5$ the solution is hole-like whereas for $\lambda>0.5$ the solution is peak-like. On Fig. 8, for three $\theta_{k B}$ angles, $\lambda$ is plotted as a function of $C_{M}$, calculated by varying the anisotropy for fixed $\beta_{\|}$. Qualitatively, the variations of $\lambda$ with $C_{M}$ depend very slightly on $\beta_{\|}$, except for the domain of validity of the solutions which is more restricted for smaller $\beta_{\|}$(see Passot et al., 2006, for detail on this issue). The model is able to reproduce the general behavior revealed by the observations: for a large angle (for instance $70^{\circ}$ ), hole solutions are obtained in the bistability region $\left(C_{M}<1\right)$ and slightly above threshold, whereas at large distance from threshold, magnetic peaks are obtained. For larger angles, solutions in mirror unstable plasmas are obtained only for large enough beta $\left(\beta_{\|}=8\right.$ at $80^{\circ}$ ) whereas for angles smaller than $\sim 60^{\circ}$ solutions exist only in unstable plasmas.

Although a MHD description appears to be sufficient to obtain a valid instability threshold and to reproduce the bistability phenomenon, retaining kinetic effects such as Landau damping and finite Larmor radius corrections is mandatory to determine the linear growth rate as well as to investigate the nonlinear development of the mirror instability. This has been achieved in further developments of the present Landau-fluid model (Passot and Sulem, 2006; Borgogno et al., 2007). A dynamical model for nonlinear mirror modes near threshold has also been recently proposed by Kuznetsov et al. (2007a) using a reductive perturbative expansion of the Vlasov-Maxwell equations. In this model, the saturation mechanism is due to local variations of the ion Larmor radius which are supplemented phenomenologically, leading to the bistability phenomenon and also the variation of the skewness with the distance to threshold. It differs from previous models for which the saturation is provided by the cooling of a population of trapped ions (Kivelson and Southwood, 1996; Pantellini, 1998).

Studying the evolution of mirror modes and the formation of coherent structures is possible by means of direct simulations of the Vlasov-Maxwell equations. We refer in particular to hybrid simulations in an expanding box (HEB code, see Hellinger and Trávníček, 2005, and references therein). The expanding box simulation models an evolution of a small fraction of the plasma which expands under the effect of the global magnetosheath flow around the magnetospheric cavity. The model replaces the spatial dependence by a temporal 


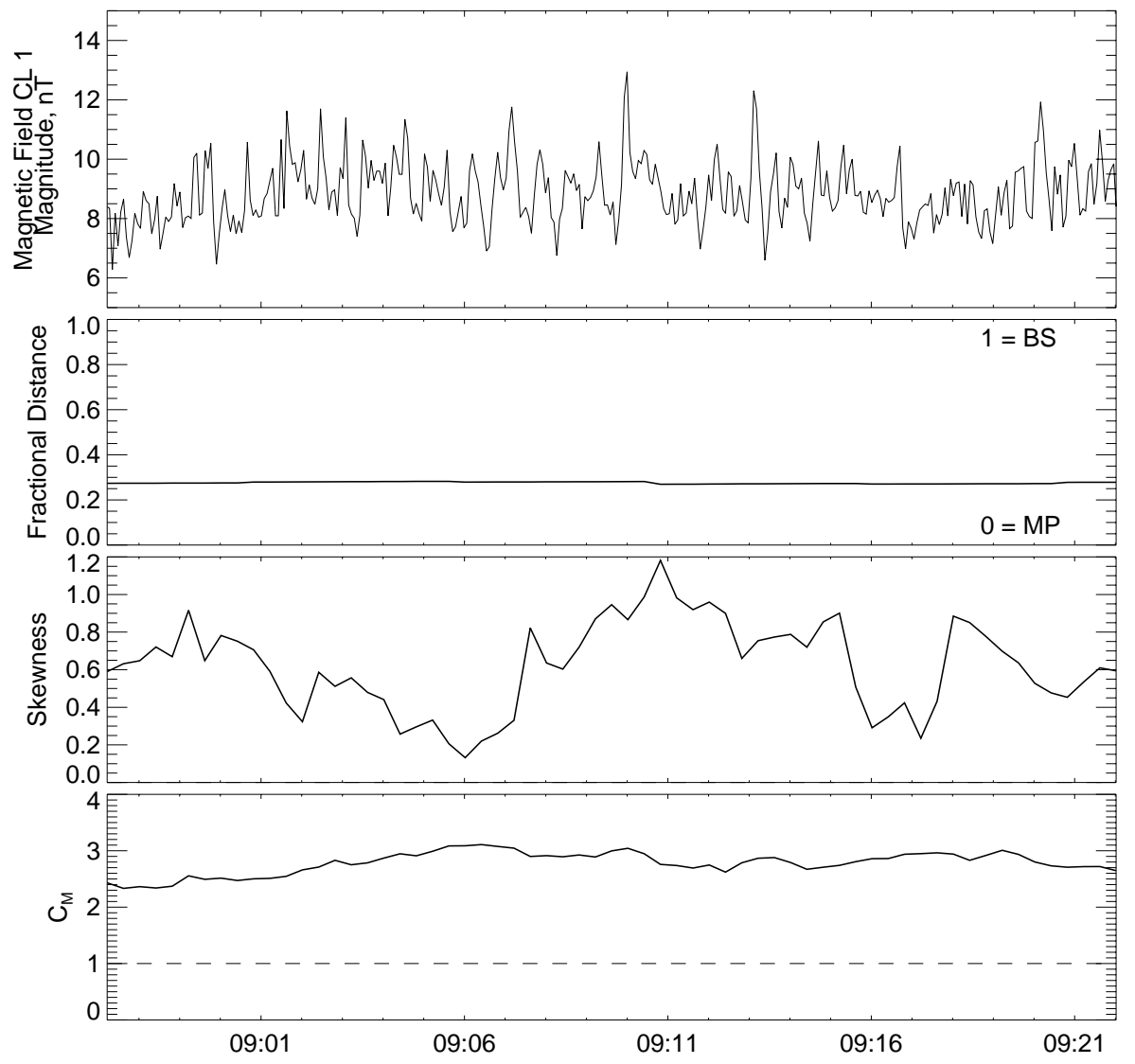

May 27, 2005

Fig. 7. Observations of magnetic peaks by Cluster 1 on 27 May 2005 (same panels as in Fig. 1).

one and neglects global inhomogeneities/heat fluxes (physical lengths increase linearly with time). In this way, it provides a self-consistent mean to study the dynamical properties of waves driven by the magnetosheath plasma.

The simulation discussed hereafter is described in Trávnícek et al. (2007); we focus here on different quantities. Figure 9 displays the evolution of the system, in the plane (skewness, $C_{M e}$ ), where the skewness is evaluated from the magnetic fluctuations in the parallel direction. Initial conditions correspond to an homogeneous plasma only weakly unstable with respect to the mirror and proton cyclotron instabilities: $T_{\perp} / T_{\|}=1.1$ and $\beta_{\|}=13\left(C_{M e}=1.4\right.$, indicated by a cross on the right panel of Figure 9). The duration of the simulation is $36000 \Omega_{p}^{-1}$ which corresponds to 36 characteristic expansion times ( $\Omega_{p}$ is the initial proton gyrofrequency). As expansion proceeds, the anisotropy increases together with mirror mode fluctuations which remain mainly sinusoidal (with thus an almost vanishing skewness) until $C_{M e} \simeq 3$. The distance to threshold increases and mirror oscillations grow in amplitude and start shaping as peaks, up to the point where the instability saturates $\left(\beta_{\|}=7, C_{M e} \simeq 3.8\right)$. Enhanced wave particle interactions induce proton isotropization which lim- its the anisotropy growth due to expansion. The system gradually reaches a marginally stable state. However, due to the expansion, $\beta$ continues to decrease, which further reduces the distance to threshold. This effect is supplemented by a gradual damping of the saturated peaks and by the formation of deeper and more isolated holes (the peak/hole transition is achieved at $\beta_{\|} \simeq 3.1, C_{M e} \simeq 1.7$ ), until the mirror threshold $C_{M e}=1$ is reached. From the analysis of Fig. 2a in Trávnícek et al. (2007), the transition from positive to negative skewness corresponds to a saturation of the mirror fluctuations. This transition is consistent with the observations of Fig. 3. When $C_{M}$ is decreased further, hole structures are damped but still survive below threshold. This behavior is also in agreement with the bistability phenomenon described earlier.

An interesting feature is that in HEB simulations, mirror fluctuations are seen for $\theta_{k B} \simeq 70^{\circ}$ (see Fig. $2 \mathrm{~b}$ in Trávnícek et al., 2007) while the ( $C_{M}$, skewness) curve in the fluid model (Fig. 8) compares favorably to both observation and simulation curves for this angle. Within the three approaches the peak-hole transition is observed for $C_{M}=1.7$ in the simulation (see Fig. 9), $C_{M}=1.5$ in the model (see Fig. 8), and $C_{M}=1.4$ in the observations (see Fig. 5 where an integration 


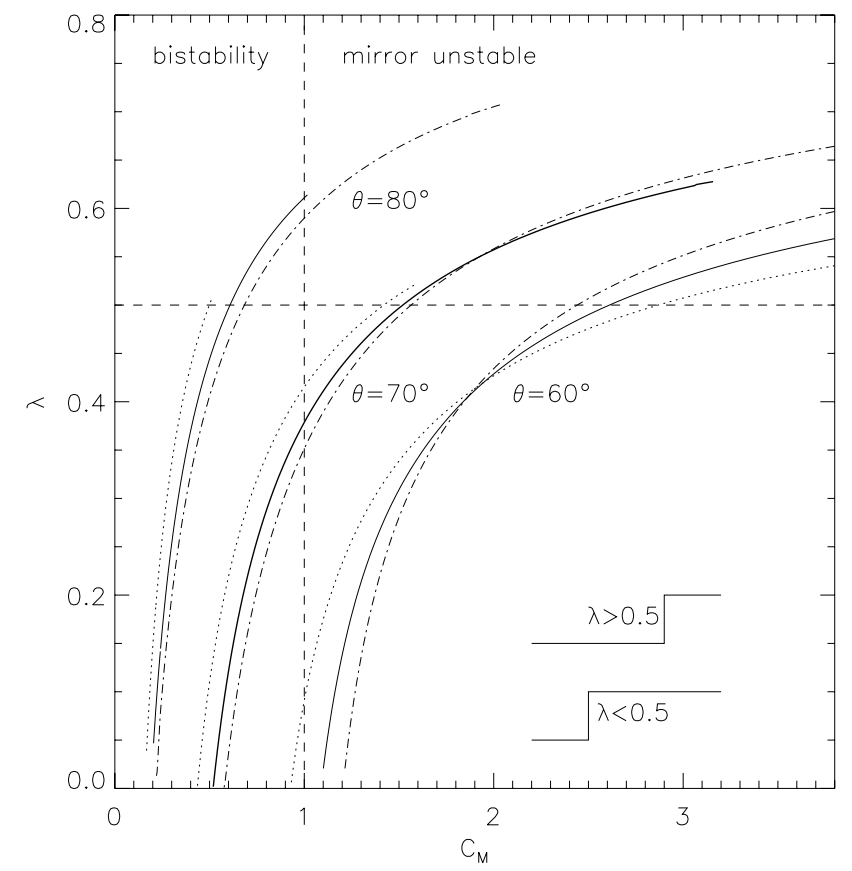

Fig. 8. Shape factor $(\lambda)$ of the solutions of Passot et al. (2006)'s fluid model, as a function of the mirror parameter $C_{M}$. As shows the insert in the lower right corner, $\lambda<0.5$ corresponds to "hole" solutions and $\lambda>0.5$ corresponds to "peak" solutions. The bistability region is in the range $0<C_{M}<1$. The different curves correspond to the validity domain of the solutions of the model for three $\theta_{k B}$ and three $\beta_{\|}$values: 2 (dotted line), 4 (solid line), and 8 (dash-dotted line). For given angle and $\beta_{\|}$values the solutions of the model live in a range of temperature anisotropy, i.e in a range of $C_{M}$ values.

over all $\theta_{k B}$ values is implicit). The three approaches thus suggest that $C_{M} \simeq 1.5$ is a significant threshold for the transition between hole and peak mirror structures. The value in excess to 1 originates from the fact that hole structures exist in a large domain of plasma conditions (not too far from threshold tough), while peaks dwell only in mirror unstable plasmas.

Califano et al. (2008) also performed simulations of the Vlasov-Maxwell equations in an extended computational domain, in the case of an homogeneous plasma. They show that it is possible to obtain hole structures similar to those obtained in the HEB model when the system is initially far from threshold. In the same paper, the persistence of large amplitude magnetic holes below threshold is observed and interpreted as as the signature of bistability.

Concerning the formation of magnetic peaks, conclusions similar to ours were proposed by Baumgärtel et al. (2003), based on hybrid simulations of the saturation of the mirror instability. This contrasts with the predictions of the theoretical models of Kivelson and Southwood (1996) and Pantellini (1998). Baumgärtel et al. (2003) suggests that this divergence might be attributed to the fact that these models assume the conservation of the magnetic moment for protons, a condition which may be violated at the scale of the mirror structures.

Finally, all three approaches share a common feature: in absolute term, extreme negative skewness values are larger than positive ones. As described in Sect. 3.3 and showed in Fig. 1, these larger values do not mean holes are deeper than peaks are high, but that holes appear in a more isolated manner than peaks. Indeed, in contrast with peaks, holes can exist in mirror stable plasma, which means they do not need particular conditions to survive.

\section{Conclusion}

In this paper, we used a statistical analysis of five years of Cluster data, supplemented by a fluid model and hybrid simulations to describe different morphological aspects of mirror structures. We use the skewness of the magnetic field fluctuations over $5 \mathrm{~min}$ windows to characterize these shapes, as negative (positive) skewness is associated with hole (peak) structures. We show that these different shapes can be related to different stages of the mirror mode dynamics which, in our analysis, is tracked by the mirror parameter $C_{M}=\beta_{\perp}\left(\frac{T_{\perp}}{T_{\|}}-1\right)$ which is equal to 1 at threshold. These approaches favorably compare regarding the existence of a bistability phenomenon, associated with a subcritical bifurcation: mirror structures in the form of magnetic holes exist below the linear threshold. This bistability phenomenon may also explain observational signatures of mirror modes in a large wavenumber regime where the growth rate is negative (Sahraoui et al., 2006). As shown by numerical simulations, the nonlinear saturation of the mirror instability leads in contrast to the formations of magnetic peaks.

In most previous analyses, the shape of the mirror structures was related to the value of the plasma $\beta$. We here show that the distance to threshold is a more relevant controlling parameter because of its direct link to the mirror mode dynamics. The skewness of mirror fluctuations are indeed correlated with $C_{M}$ : deep holes, due to the bistability process, are observed for mirror stable conditions $\left(C_{M}<1\right)$, sinusoidal mirror modes and moderate holes and peaks are encountered near threshold $C_{M} \simeq 1.5$, whereas large peaks, are obtained far from threshold $C_{M} \gtrsim 2$. Because they are present in unstable plasmas, peaks are generally observed in groups whereas holes can appear isolated. This is illustrated by the skewness magnitudes which are, in all the three approaches, significantly larger (in absolute value) for holes than for peaks. The location of the mirror structures in the magnetosheath is tracked by computing the fractional distance $F$ between the shock $(F=1)$ and the magnetopause $(F=0)$. In agreement with other studies (Joy et al., 2006; Soucek et al., 2008), we find that peaks are mostly observed in the middle of the magnetosheath whereas most holes are observed close to the magnetopause. This is also in agreement with hybrid simulations which mimic the magnetosheath plasma condition 

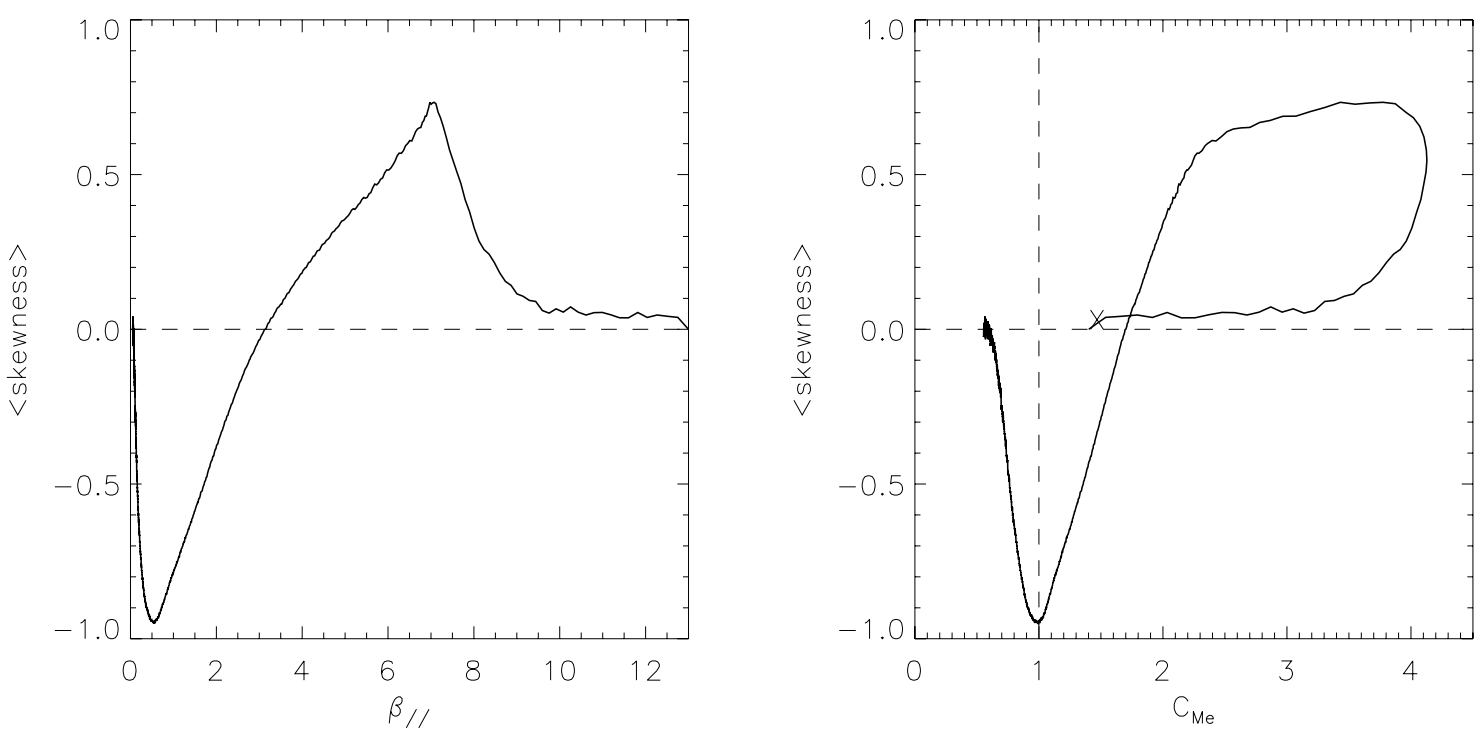

Fig. 9. Results of the HEB simulation code. On the left panel the skewness of parallel magnetic fluctuations as a function of proton $\beta_{\|}$is displayed. The time evolves from high $\beta_{\|}$values to lower ones whereas the anisotropy increases, both processes being due to the forced expansion of the plasma box modeled in the code. The transition from positive to negative skewness occurs for $\beta_{\|} \simeq 3.1$. On the right panel the skewness is plotted as a function of $C_{M e}\left(\beta_{e}=1\right)$ and the starting point of the simulation is referenced by the cross.

evolution from the shock (large $\beta$, moderate anisotropy) to the magnetopause (small $\beta$, large anisotropy).

From the combined views of observations and models we can propose the following scenario for the evolution of mirror structures. Large peak structures grow out from moderately unstable plasma (typically observed behind the bow shock), reaching saturation at sufficiently large distance from threshold (in the middle magnetosheath depending on the convection time), and, as $\beta$ is further decreased closer to the magnetopause, the plasma turns to be mirror stable, which is accompanied by a decay of the peaks to the profit of hole structures which can survive these conditions. Close to the magnetopause, trains of magnetic holes with very small values of the magnetic field amplitude can modify the reconnection rate. Further down the magnetosheath flanks, the analysis of Soucek et al. (2008) showed that mostly magnetic holes are observed even at larger magnetopause distance, due to a gradual decrease in temperature anisotropy.

On the observational point of view, a challenge is posed by recent theoretical works Califano et al. (2008) which predict, near threshold, a flattening of the distribution functions at small parallel velocities (corresponding to resonant particles). Precise measurements at small energies are then required to capture this effect due to a diffusion in velocity space. Another interesting development that multi-spacecraft observations can achieve, would be to observe the same structure in different stages of evolution in order to study the mirror dynamics, as in the numerical simulations.
Acknowledgements. This work was performed in the frame of the International Space Science Institute meetings of Team 80 "The effect of ULF turbulence and flow chaotisation on plasma energy and mass transfer at the magnetopause". V.G. thanks the CDPP team members for their efforts in developing the analysis tools used in this study. P.H. and P.T. acknowledge the Czech grants GAAV IAA300420702 and IAA300420602. The hybrid simulation has been performed on Amalka supercomputing facility at IAP, ASCR.

Topical Editor I. A. Daglis thanks two anonymous referees for their help in evaluating this paper.

\section{References}

Anderson, B. J., Fuselier, S. A., Gary, S. P., and Denton, R. E.: Magnetic spectral signatures in the Earths magnetosheath and plasma depletion layer, J. Geophys. Res., 99(A4), 5877-5892, doi:10.1029/93JA02827, 1994.

Balikhin, M. A., Pokhotelov, O. A., Walker, S. N., Amata, E., Andre, M., Dunlop, M., and Alleyne, H. S. C. K.: Minimum variance free wave identification: Application to Cluster electric field data in the magnetosheath, Geophys. Res. Lett., 30(10), 1508, doi:10.1029/2003GL016918, 2003.

Balogh, A., Carr, C. M., Acuña, M. H., et al.: The Cluster Magnetic Field Investigation: overview of in-flight performance and initial results, Ann. Geophys., 19, 1207-1217, 2001, http://www.ann-geophys.net/19/1207/2001/.

Baumgärtel, K., Dubinin, E., Sauer, K., and Story, T. R.: Solar wind magnetic holes: signature of slow mode type MHD solitons?, Adv. Space Res., 20, 69-74, 1997.

Baumgärtel, K.: Soliton approach to magnetic holes, J. Geophys. Res., 104, 28295-28308, 1999. 
Baumgärtel, K.: Fluid approach to mirror mode structures, Planet. Space. Sci., 49, 1239-1247, 2001.

Baumgärtel K., Sauer, K., and Dubinin, E.: Towards understanding magnetic holes: Hybrid simulations, Geophys. Res. Lett., 30(14), 1761, doi:10.1029/2003GL017373, 2003.

Bavassano Cattaneo, M. B., Basile, C., Moreno, G., and Richardson, J. D.: Evolution of mirror structures in the magnetosheath of Saturn from the bow shock to the magnetopause, J. Geophys. Res., 103, 11961-11972, 1998.

Borgogno, D., Passot, T., and Sulem, P. L.: Magnetic holes in plasmas close to the mirror instability, Nonlin. Processes Geophys., 14, 373-383, 2007, http://www.nonlin-processes-geophys.net/14/373/2007/.

Burlaga, L. F., Ness, N. F., and Acũna, M. H.: Trains of magnetic holes and magnetic humps in the heliosheath, Geophys. Res. Lett., 33, L21106, doi:10.1029/2006GL027276, 2006.

Burlaga, L. F., Ness, N. F., and Acuna, M. H.: Linear magnetic holes in a unipolar region of the heliosheath observed by Voyager 1, J. Geophys. Res., 112, A07106, doi:10.1029/2007JA012292, 2007.

Califano, F., Hellinger, P., Kuznetsov, E., Passot, T., Sulem, P. L., and Travnicek, P.: Nonlinear mirror mode dynamics: simulations and modeling, J. Geophys. Res., 113, A08219 doi:10.1029/2007JA012898, 2008.

Chisham, G., Schwartz, S. J., Balikhin, M. A., and Dunlop, M. W.: AMPTE observations of mirror mode waves in the magnetosheath: Wavevector determination, J. Geophys. Res., 104(A1), 437-448, doi:10.1029/1998JA900044, 1999.

Constantinescu, O. D.: Self-consistent model for mirror structures, J. Atmos. Terr. Phys., 64, 645-649, 2002

Denton, R. E., Gary, S. P., Anderson, B. J., LaBelle, J. W., and Lessard, M.: Low-frequency fluctuations in the magnetosheath near the magnetopause, J. Geophys. Res., 100(A4), 5665-5680, doi:10.1029/94JA03024, 1995.

Erdös, G. and Balogh, A.: Statistical properties of mirror mode structures observed by Ulysses in the magnetosheath of Jupiter, J. Geophys. Res., 101, 1-12, 1996.

Fazakerley, A. N. and Southwood, D. J.: Mirror instability in the magnetosheath, Adv. Space Res., 14(7), 65-68, 1994.

Fuselier, S. A., Anderson, B. J., Gary, S. P., and Denton, R. E.: Inverse correlations between the ion temperature anisotropy and plasma beta in the Earths quasi-parallel magnetosheath, J. Geophys. Res., 99, 14931-14936, doi:10.1029/94JA00865, 1994.

Gary, S. P. and Lee, M. A.: The ion cyclotron anisotropy instability and the inverse correlation between proton anisotropy and proton beta, J. Geophys. Res., 99(A6), 11297-11302, doi:10.1029/94JA00253, 1994

Gary, S. P., Fuselier, S. A., and Anderson, B. J.: Ion anisotropy instabilities in the magnetosheath, J. Geophys. Res., 98(A2), 14811488, doi:10.1029/92JA01844, 1993.

Gary, S. P., Lavraud, B., Thomsen, M. F., Lefebvre, B., and Schwartz, S. J.: Electron anisotropy constraint in the magnetosheath: Cluster observations, Geophys. Res. Lett., 32, L13109, doi:10.1029/2005GL023234, 2005.

Génot, V., Schwartz, S. J., Mazelle, C., Balikhin, M., Dunlop, M., and Bauer, T. M.: Kinetic study of the mirror mode, J. Geophys. Res., 106(A10), 21611-21622, doi:10.1029/2000JA000457, 2001.

Génot, V.: Mirror and firehose instabilities in the heliosheath, The
Astrophys. J., 687, L119-L122, 2008.

Génot, V., Budnik, E., Jacquey, C., Dandouras, I., and Lucek, E.: Mirror modes observed with Cluster in the Earth's magnetosheath : statistical study and IMF/solar wind dependence, Adv. Geosci., in press, 2009.

Hall, A. N.: Finite ion Larmor radius modifications to the firehose and mirror instabilities, J. Plasma Phys., 21, 431-443, 1979.

Hellinger P., Trávníček, P., Mangeney, A., and Grappin, R.: Hybrid simulations of the magnetosheath compression: Marginal stability path, Geophys. Res. Lett., 30(18), 1959, doi:10.1029/2003GL017855, 2003.

Hellinger P. and Trávníček, P.: Magnetosheath compression: Role of characteristic compression time, alpha particle abundance, and alpha/proton relative velocity, J. Geophys. Res., 110, A04210, doi:10.1029/2004JA010687, 2005.

Hellinger P.: Comment on the linear mirror instability near the threshold, Phys. Plasmas, 14, 8, 2007.

Horbury, T. S., Lucek, E. A., Balogh, A., Dandouras, I., and Rème, H.: Motion and orientation of magnetic field dips and peaks in the terrestrial magnetosheath, J. Geophys. Res., 109, 9209, doi:10.1029/2003JA010237, 2004.

Joy, S. P., Kivelson, M. G., Walker, R. J., Khurana, K. K., Russell, C. T., and Paterson, W. R.: Mirror mode structures in the Jovian magnetosheath, J. Geophys. Res., 111, A12212, doi:10.1029/2006JA011985, 2006.

Kaufmann, R. L., Horng, J.-T., and Wolfe, A.: Large-amplitude hydromagnetic waves in the inner magnetosheath, J. Geophys. Res., 75(25), 4666-4676, 1970.

Kivelson, M. and Southwood, D.: Mirror instability II: The mechanism of non-linear saturation, J. Geophys. Res., 101, 1736517371, 1996.

Kuznetsov, E. A., Passot, T., and Sulem, P. L.: Dynamical model for nonlinear mirror modes near threshold, Phys. Rev. Lett., 98(23), 235003, 2007a.

Kuznetsov, E. A., Passot, T., and Sulem, P. L.: Nonlinear theory of mirror instability near threshold, Pis'ma v ZhETF, 86, 725-730, JETP Lett., 86, 637-642, 2007b.

Leckband, J. A., Burgess, D., Pantellini, F. G. E., and Schwartz, S J.: Ion distributions associated with mirror waves in the Earth's magnetosheath, Adv. Space Res., 15(8-9), 345-348, 1995.

Lin, C.-H., Chao, J. K., Lee, L. C., Wu, D. J., Li, Y., Wu, B. H., and Song, P.: Identification of mirror waves by the phase difference between perturbed magnetic field and plasmas, J. Geophys. Res., 103(A4), 6621-6632, doi:10.1029/97JA03474, 1998.

Liu, Y., Richardson, J. D., Belcher, J. W., Kasper, J. C., and Skoug, R. M.: Plasma depletion and mirror waves ahead of interplanetary coronal mass ejections, J. Geophys. Res., 111, A09108, doi:10.1029/2006JA011723, 2006.

Lucek, E. A., Dunlop, M. W., Balogh, A., Cargill, P., Baumjohann, W., Georgescu, E., Haerendel, G., and Fornacon, G.-H.: Identification of magnetosheath mirror modes in Equator-S magnetic field data, Ann. Geophys., 17, 1560-1573, 1999, http://www.ann-geophys.net/17/1560/1999/.

Lucek, E. A., Dunlop, M. W., Horbury, T. S., Balogh, A., Brown, P., Cargill, P., Carr, C., Fornaçon, K.-H., Georgescu, E., and Oddy, T.: Cluster magnetic field observations in the magnetosheath: four-point measurements of mirror structures, Ann. Geophys., 19, 1421-1428, 2001 http://www.ann-geophys.net/19/1421/2001/. 
Masood, W. and Schwartz, S. J.: Observations of the development of electron temperature anisotropies in Earths magnetosheath, J. Geophys. Res., 113, A01216, doi:10.1029/2007JA012715, 2008.

Pantellini, F. G. E.: A model of the formation of stable nonpropagating magnetic structures in the solar wind based on the nonlinear mirror instability, J. Geophys. Res., 103, 4789-4798, 1998.

Passot T. and Sulem, P. L.: A fluid model with finite Larmor radius effects for mirror mode dynamics, J. Geophys. Res., 111, A04203, doi:10.1029/2005JA011425, 2006.

Passot T., Ruban, V., and Sulem, P. L.: Fluid description of trains of stationary mirror structures in a magnetized plasma, Phys. Plasmas, 13, 102310-102310-10, 2006.

Pokhotelov, O. A., Treumann, R. A., Sagdeev, R. Z., Balikhin, M. A., Onishchenko, O. G., Pavlenko, V. P., and Sandberg, I.: Linear theory of the mirror instability in nonMaxwellian space plasmas, J. Geophys. Res., 107(A10), 1312, doi:10.1029/2001JA009125, 2002.

Rème, H., Aoustin, C., Bosqued, J. M., et al.: First multispacecraft ion measurements in and near the Earths magnetosphere with the identical Cluster ion spectrometry (CIS) experiment, Ann. Geophys., 19, 1303-1354, 2001, http://www.ann-geophys.net/19/1303/2001/.

Sahraoui, F., Belmont, G., Rezeau, L., Cornilleau-Wehrlin, N., Pincon, J. L., and Balogh, A.: Anisotropic turbulent spectra in the terrestrial magnetosheath as seen by the cluster spacecraft, Phys. Rev. Lett.,96(7), 075002, 2006.

Schwartz, S. J., Burgess, D., and Moses, J. J.: Low-frequency waves in the Earth's magnetosheath: present status, Ann. Geophys., 14, 1134-1150, 1996, http://www.ann-geophys.net/14/1134/1996/.

Shapiro, V. D. and Shevchenko, V. I.: Quasilinear theory of instability of a plasma with an anisotropic ion velocity distribution, Sov. Phys. JETP 18, Engl. Transl., 1109-1116, 1964.

Shue, J.-H., Chao, J. K., Fu, H. C., Russell, C. T., Song, P., Khurana, K. K., and Singer, H. J.: A new functional form to study the solar wind control of the magnetopause size and shape, J. Geophys. Res., 102(5), 9497-9512, 1997.

Song, P., Russell, C. T., and Gary, S. P.: Identification of lowfrequency fluctuations in the terrestrial magnetosheath, J. Geophys. Res., 99(A4), 6011-6026, doi:10.1029/93JA03300, 1994.

Sonnerup, B. U. O., and Cahill Jr., L. J.: Magnetopause structure and attitude from Explorer 12 observations, J. Geophys. Res., 72, 171-183, 1967.
Soucek J., Lucek, E., and Dandouras, I.: Properties of magnetosheath mirror modes observed by Cluster and their response to changes in plasma parameters, J. Geophys. Res., 113, A04203, doi:10.1029/2007JA012649, 2008.

Sperveslage, K., Neubauer, F. M., Baumgärtel, K., and Ness, N. F.: Magnetic holes in the solar wind between $0.3 \mathrm{AU}$ and $17 \mathrm{AU}$, Nonlin. Processes Geophys., 7, 191-200, 2000, http://www.nonlin-processes-geophys.net/7/191/2000/.

Stasiewicz, K.: Reinterpretation of mirror modes as trains of slow magnetosonic solitons, Geophys. Res. Lett., 31(21), L21804, doi:10.1029/2004GL021282, 2004a.

Stasiewicz, K.: Theory and observations of slow-mode solitons in space plasmas, Phys. Rev. Lett., 93(12), 125004, $2004 \mathrm{~b}$.

Stix, T. H.: The theory of plasma waves, McGraw-Hill, New York, 1962.

Tátrallyay, M. and Erdös, G.: The evolution of mirror mode fluctuations in the terrestrial magnetosheath, Planet. Space Sci., 50, 593-599, 2002.

Tátrallyay, M. and Erdös, G.: Statistical investigation of mirror type magnetic field depressions observed by ISEE-1, Planet. Space Sci., 53, 33-40, 2005.

Trávnícek, P., Hellinger, P., Taylor, M. G. G. T., Escoubet, C. P., Dandouras, I., and Lucek, E.: Magnetosheath plasma expansion : hybrid simulations, Geophys. Res. Lett., 34(15), L15104, doi:10.1029/2007GL029728, 2007.

Verigin, M. I., Tátrallyay, M., Erdös, G., and Kotova, G. A.: Magnetosheath-Interplanetary medium reference frame: Application for a statistical study of mirror type waves in the terrestrial plasma environment, Adv. Space Res., 37, 515-521, 2006.

Walker, S. N., Balikhin, M. A., and Dunlop, M.: Mirror structures in the magnetosheath: 3D structures on plane waves, Adv. Space Res., 30, 2745-2750, 2002.

Winterhalter, D., Neugebauer, M., Goldstein, B. E., Smith, E. J., Bame, S. J., and Balogh, A.: Ulysses field and plasma observations of magnetic holes in the solar wind and their relation to mirror-mode structures, J. Geophys. Res., 99, 23371-23381, 1994. 